\title{
Roughness measurement of 2D curvilinear patterns: challenges and advanced methodology
}

\author{
Jonathan Pradelles ${ }^{\mathrm{a}}$, Loïc Perraud ${ }^{\mathrm{a}}$, Aurélien Fay ${ }^{\mathrm{a}}$, Jean-Baptiste Henry ${ }^{\mathrm{a}}$, Jessy Bustos ${ }^{\mathrm{a}}$, \\ Estelle Guyez ${ }^{\mathrm{a}}$, Sébastien Berard-Bergery ${ }^{\mathrm{a}}$, Aurélie Le Pennec ${ }^{\mathrm{a}}$, Mohamed Abaidi ${ }^{\mathrm{b}}$, Jordan \\ Belissard $^{\mathrm{b}}$, Nivea Schuch ${ }^{\mathrm{b}}$, Thiago Figueiro ${ }^{\mathrm{b}}$, Matthieu Millequant ${ }^{\mathrm{b}}$, Patrick Schiavone ${ }^{\mathrm{b}, \mathrm{c}}$ \\ a Univ. Grenoble Alpes, CEA, LETI, DPFT, Lithography Laboratory, F-38000 Grenoble, France \\ ${ }^{\mathrm{b}}$ ASELTA Nanographics, 4 pl. Robert Schuman, F-38000 Grenoble, France \\ ${ }^{c}$ Univ. Grenoble Alpes, CNRS, CEA/LETI-Minatec, Grenoble INP, LTM, F-38054 Grenoble-France
}

The importance of measurement and analysis of pattern roughness in SEM (Scanning Electron Microscope) images has continuously grown in the past few decades since it affects performances of devices [1]. In parallel, non-Manhattan patterns - such as curvilinear and shapes with multiple angles are more and more present in recent lithography landscape [2], [3]. Key examples are photonic devices, ILT (Inverse Lithography Technology) patterns in photomask fabrication, and DSA (Directed SelfAssembly) fingerprint patterns for early material development. In this article the challenges associated with roughness measurement on such curvilinear patterns will be presented and algorithms and methodology to tackle these challenges will be proposed.

The proposed methodology relies on robust-to-noise contour extraction algorithms [4]. In this paper, an original method to evaluate and validate contour extraction algorithms, in the context of roughness measurement, is proposed. The schematic workflow is illustrated in Figure 1. Both a SEM model and a noise model are calibrated using experimental SEM images from state-of-the-art equipment and processes. This allows the generation of synthetic SEM images with arbitrary layout, programmed roughness and controlled noise (equivalent to 'frame by frame' SEM image acquisition). Edge detection algorithms and advanced 'PSD analysis' are applied to the images for roughness measurement. Same strategy may then be applied to 'real' SEM images.

The method is first carried out in 1D patterns (straight lines). For that, synthetic SEM images are generated, following the protocol of ref [5]. From this step, an excellent correlation with the results obtained in [6] is found. Additionally, to that, a very low noise sensitivity of the edge contour extraction algorithm is noticed (Figure 2).

The methodology is then extended to 2D patterns. As a first step, we make use of synthetic SEM images to discuss the potential problems raised by curvilinear patterns, such as reference determination, data interpolation and closing contours. In the same way as for 1D patterns, a programmed roughness, entered as input in the workflow of Figure 1, is found back in the analysis after contour extraction and PSD analysis. Using synthetic images for different number of frames, the contour extraction sensitivity to noise can also be explored.

Finally, the methodology is successfully applied to experimental SEM images: unbiased PSD of 2D curved patterns are determined for two classes of applications: photonic devices (as shown in Figure 3) and DSA fingerprint (as shown in Figure 4).

Keywords: 2D roughness metrology, curvilinear patterns, contour metrology, PSD analysis, SEM model, synthetic images 


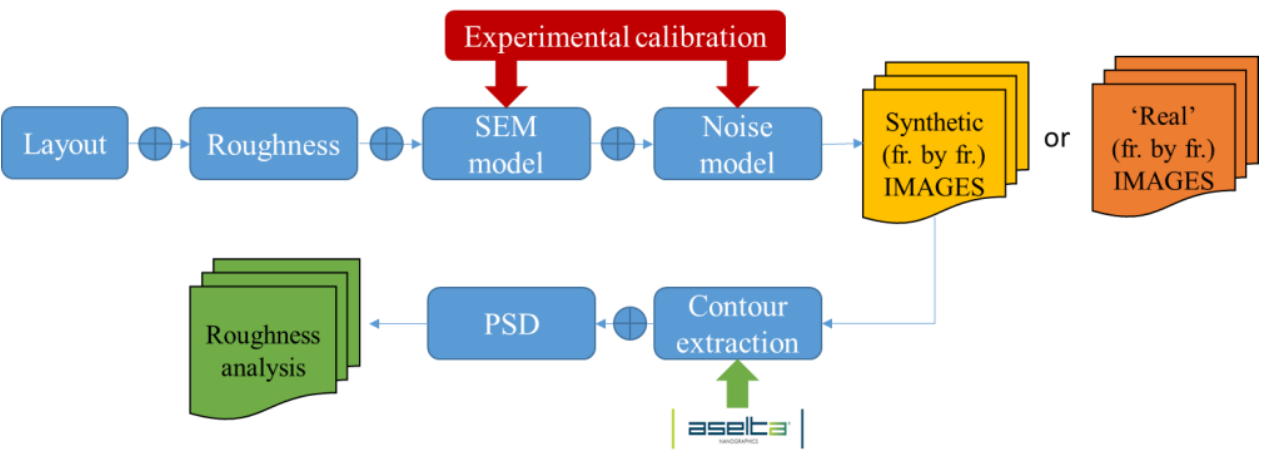

Figure 1: Schematic workflow used to measure roughness on curvilinear 2D patterns.

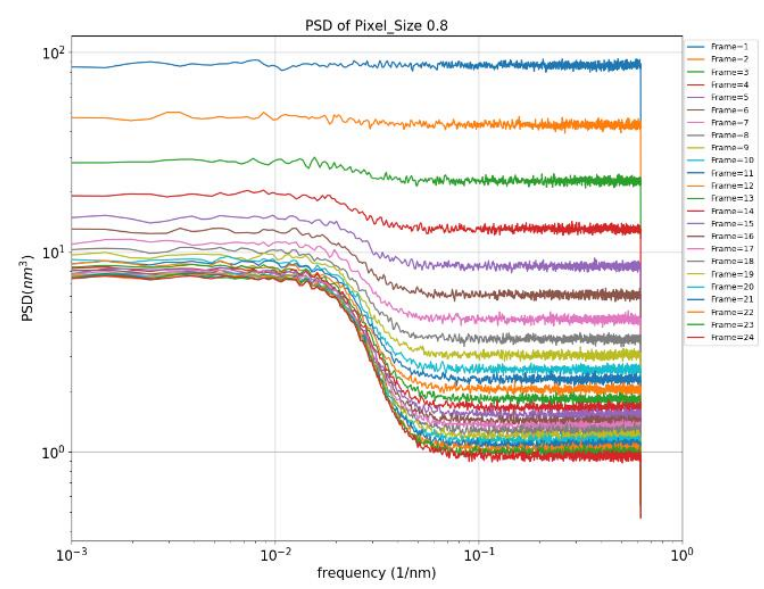

Figure 2: 1D-pattern roughness measurement following protocol of [5] with excellent correlation with results of [6].

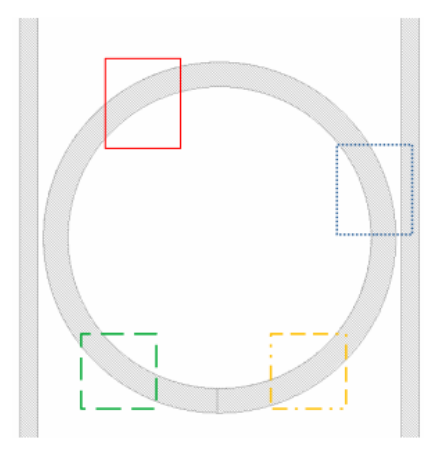

(a)
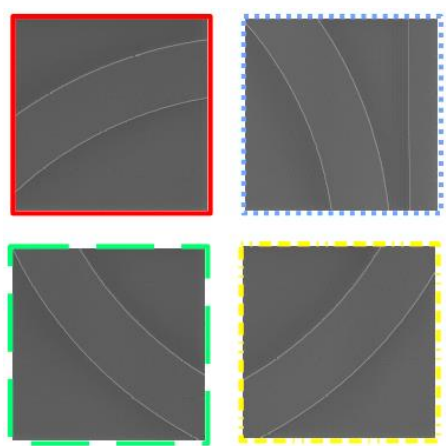

(b)

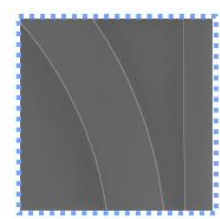

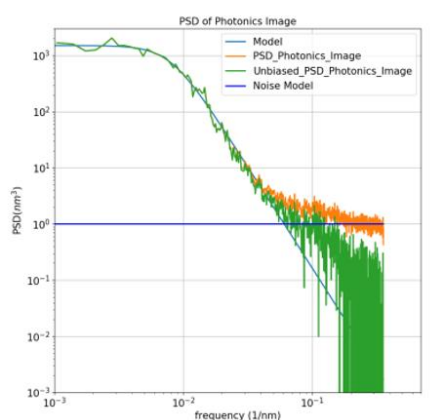

(c)

Figure 3: (a), a ring oscillator design from a photonic device. (b) a detailed zoom of the associated SEM image, showing the variability of angles that have to be considered for the roughness measurement. (c), the unbiased PSD for the ring oscillator in (a). 


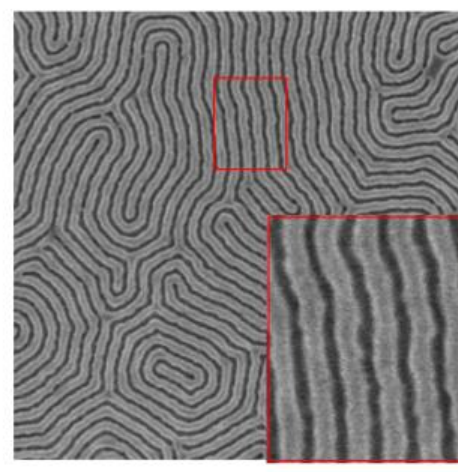

(a)

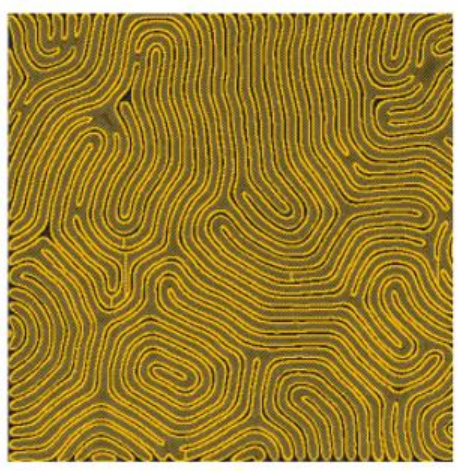

(b)

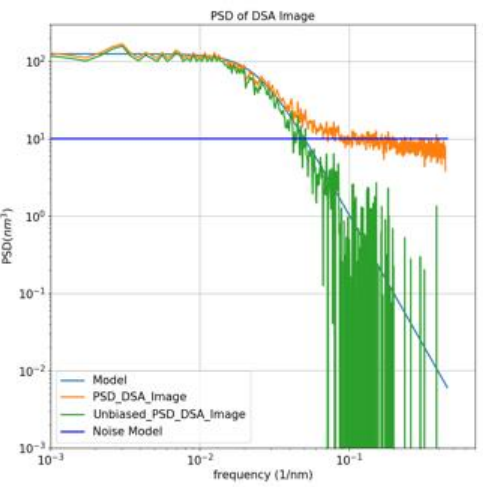

(c)

Figure 4: (a) a SEM image from a DSA fingerprint and zoom in detailed (highlighted in red). (b), the result of the contour extraction [4], and (c), the associated unbiased PSD.

\section{REFERENCES}

[1] P. Oldiges, Qimghuamg Lin, K. Petrillo, M. Sanchez, M. Ieong and M. Hargrove, "Modeling line edge roughness effects in sub 100 nanometer gate length devices," 2000 International Conference on Simulation Semiconductor Processes and Devices (Cat. No.00TH8502), Seattle, WA, USA, 2000, pp. 131-134, doi: 10.1109/SISPAD.2000.871225.

[2] L.Dalton, "Photonic integration improves on current technologies", 7 October 2007, SPIE Newsroom. doi: $10.1117 / 2.1200710 .0866$

[3] C.J. Koh, P. Madden, "Manhattan or non-Manhattan?: a study of alternative VLSI routing architectures", Proceedings of the 10th Great Lakes symposium on VLSIMarch 2000 Pages 47-52, doi: $10.1145 / 330855.330961$

[4] Le-Gratiet, B., Bouyssou, R., Ducote, J., Ostrovsky, A., Beylier, C., Gardin, C., Schuch, N. G., Annezo, V., Schneider, L., Millequant, M., Petroni, P., Figueiro, T. and Schiavone, P., "Contour based metrology: "make measurable what is not so"," Metrology, Inspection, and Process Control for Microlithography XXXIV, SPIE (2020).

[5] Gian Francesco Lorusso, Takumichi Sutani, Vito Rutigliani, Frieda van Roey, Alain Moussa, Anne-Laure Charley, Chris Mack, Patrick Naulleau, Chami Perera, Vassilios Constantoudis, Masami Ikota, Toru Ishimoto, and Shunsuke Koshihara "Need for LWR metrology standardization: the imec roughness protocol," Journal of Micro/Nanolithography, MEMS, and MOEMS 17(4), 041009 (12 September 2018). https://doi.org/10.1117/1.JMM.17.4.041009

[6] Chris A. Mack, Frieda Van Roey, and Gian F. Lorusso "Unbiased roughness measurements: subtracting out SEM effects, part 3", Proc. SPIE 10959, Metrology, Inspection, and Process Control for Microlithography XXXIII, 109590P (26 March 2019); https://doi.org/10.1117/12.2515898 